ORIGINAL ARTICLE

\title{
A simple and rapid approach for human herpesvirus type 8 subtype characterization using single base extension
}

\author{
M.L. Hulaniuk ${ }^{1}$, D. Corach ${ }^{2,3}$, J. Trinks ${ }^{1,2}$ (D) and M. Caputo ${ }^{2,3}$ \\ 1 Instituto de Medicina Traslacional e Ingeniería Biomédica (IMTIB), CONICET, Instituto Universitario del Hospital Italiano (IUHI), Hospital Italiano \\ (HIBA), Buenos Aires, Argentina \\ 2 Consejo Nacional de Investigaciones Científicas y Técnicas (CONICET), Buenos Aires, Argentina \\ 3 Universidad de Buenos Aires, Facultad de Farmacia y Bioquímica, Departamento de Microbiología, Inmunología, Biotecnología y Genética, \\ Cátedra de Genética Forense y Servicio de Huellas Digitales Genéticas, Buenos Aires, Argentina
}

Significance and Impact of the Study: In this study, we developed and validated a single base extension method for characterization of HHV-8 ORFK1 subtypes. This assay is simple, rapid, easy to perform, reliable and cost-effective compared to the 'gold standard' method. This technique can be adapted to other viral genomes and be used as an efficient screening method for genotyping in a single tube, rapidly and with easy data interpretation.

\section{Keywords}

Kaposi's sarcoma-associated herpesvirus (human herpesvirus type 8), ORFK1,

phylogenetic analysis, single base extension, subtypes.

\section{Correspondence}

Julieta Trinks, Instituto de Medicina Traslacional e Ingeniería Biomédica (IMTIB), CONICET, Instituto Universitario del Hospital Italiano (IUHI), Hospital Italiano (HIBA), Potosí 4240, Buenos Aires, C1199ACL, Argentina. E-mail: julieta.trinks@hospitalitaliano.org.ar

Julieta Trinks and Mariela Caputo contributed equally to this study.

2021/0321: received 12 February 2021, revised 20 May 2021 and accepted 21 May 2021

doi:10.1111/lam.13515

\begin{abstract}
Sequence analysis of the ORFK1 of human herpesvirus type 8 (HHV-8) allows the identification of six major subtypes $(\mathrm{A}-\mathrm{F})$, which are related to human migrations and the clinical progression of Kaposi's sarcoma. Sequencing and subsequent phylogenetic analysis of ORFK1 is considered to be the most reliable method for HHV-8 genotyping. However, it exhibits challenges and limitations. Herein, we designed and validated a single base extension (SBE) protocol for characterization of HHV-8 ORFK1 subtypes. A nested polymerase chain reaction (PCR) protocol was carried out to amplify a small 294-bp PCR product encompassing four single nucleotide polymorphisms at positions 360 , 406, 465 and 527 of the HHV-8 genome. Finally, a multiplex SBE technique was developed and validated in 20 samples previously genotyped by phylogenetic analysis. The patterns obtained in this reaction could successfully discriminate between ORFK1 subtypes. The typing results obtained completely matched with those of the 'gold standard' method in all analysed samples. This method can reliably identify HHV-8 subtypes A, B and C, which are the most prevalent ones worldwide, and the remaining subtypes (D, E and F). SBE can be useful as an efficient, rapid and low-cost screening method for viral genotyping in a single tube, particularly samples with low-quality DNA, and with easy data interpretation.
\end{abstract}

\section{Introduction}

As the cornerstone of molecular epidemiology, an effective molecular typing scheme should provide sufficient discriminatory power, be reproducible among different laboratories, and be easily performed and standardized (Ranjbar et al. 2014).

To date, sequencing and subsequent phylogenetic analysis is the most reliable method for the correct identification of viral subtypes and subclades. In the clinical settings, viral genotype assignment is necessary to infer prognosis and treatment response, as well as to monitor and track strains related to drug and/or vaccine resistance, with increased virulence or transmissibility (Venner et al. 2016; Lin and Kao 2017; Abecasis et al. 2018). Furthermore, phylogenetic analysis has been valuable as a forensic tool for transmission networks and outbreak and epidemic investigations (Cella et al. 2018; Ciotti et al. 2019), and it can also be used to recognize the evolutionary history of a viral genotype in a given geographical 
area and the estimated time of the most common recent ancestor (Bons and Regoes 2018).

However, this viral genotyping method exhibits serious limitations. In addition to ambiguous results when using conserved or similar genes due to horizontal gene transfer between viruses, gene duplication and gene capture from the host (Young and Gillung 2019), the most important and common challenge encountered is that of the requirement of a high-quality DNA template and an amplified long PCR fragment for high accuracy (Ganesh and Zhang 2019). This drawback determines that phylogenetic analysis could be not feasible in all sample types and for highly variable viral genomic regions (Wohl et al. 2016; Kenmoe et al. 2020).

This is the case for human herpesvirus type 8 (HHV8), a member of the gamma-herpesvirinae known for its role in the development of all clinical-epidemiological forms of Kaposi's sarcoma (KS) and several lymphoproliferative disorders (Li et al. 2017).

HHV-8 is characterized by its high genetic variability across the entire genome, with the highest level of genetic variation observed at the $5^{\prime}$ and $3^{\prime}$ ends of the genome. Sequence analysis of the highly variable open reading frame (ORF) K1 region has allowed the identification of six major subtypes of $\mathrm{HHV}-8$, named A-F. Moreover, several subclades were described for subtypes A-E (Kajumbula et al. 2006; Cassar et al. 2012; Pérez and Tous 2017). Geographically, HHV-8 subtypes show distinct distribution patterns related to the coevolution of the viruses with human beings during migrations along the history (Hayward and Zong 2007). In addition, evidence of a relationship between the clinical progression of $\mathrm{KS}$ and HHV-8 subtypes has also been proposed. In this regard, subtype A was significantly associated with fast progression of classic KS and with high viral load, whereas subtype $\mathrm{C}$ was related to low viral load and slow KS progression and subtype $\mathrm{B}$ has been linked to a better prognosis of this disease (Mancuso et al. 2008; CordialiFei et al. 2015; Tozetto-Mendoza et al. 2016).

Genotyping HHV-8 could be challenging. First, complete or even partial ORFK1 nested PCR amplification is often not possible in all HHV-8 DNA samples (Ötvös et al. 2014; Marashi et al. 2018). Around 20-80\% of successful ORFK1 amplification has been reported, probably as a consequence of the high genetic variability of this genomic region, and the low viral load detected in samples obtained during latent infection (Zhang et al. 2008; Hulaniuk et al. 2020; Jary et al. 2020). Therefore, several studies have resorted to the use of multiple nested PCR protocols to increase amplification rates. Another possible explanation for this methodological challenge is the fact that formalin-fixed paraffin-embedded (FFPE) KS tissues are a major source of HHV-8 DNA used in molecular epidemiology studies. Unfortunately, the FFPE process causes fragmentation and chemical modification in DNA, such as cross-linking between protein and DNA, deamination and adducts (Do and Dobrovic 2015). Such modifications result in loss of quality and number of amplifiable DNA templates and pose significant challenges to PCR efficiency (Mubemba et al. 2017). In addition, the average-size DNA that is extracted from FFPE tissues has been reported as 300-400 base pairs (bp) which represents a problem not only for PCR amplification but also for genotyping by means of phylogenetic analysis (Kocjan et al. 2016; Mubemba et al. 2017). In conclusion, these drawbacks affect the reproducibility and standardization of this genotyping method and, as a consequence, turn it into a time-consuming and difficult-to-perform approach in clinical samples.

To overcome these limitations, other easier and faster molecular assays that identify single nucleotide polymorphisms (SNPs) are being used to type viral strains (Rendón et al. 2015; Irshad et al. 2016). Single base extension technique is one of the methods used to identify SNPs (Sobrino et al. 2005). Various multiplex SBE assays have been validated successfully for the analysis of mitochondrial DNA, autosomes, the Y-chromosome, Duffy and $\mathrm{ABO}$ blood groups, bacteria and microbial eukaryotes (Araujo et al. 2015; Zeddeman et al. 2015; Chen et al. 2019; Peng et al. 2020), and viruses (Webster et al. 2009; Naidu et al. 2012; Arpan et al. 2016; Mou et al. 2019). However, to the best of our knowledge, the SBE-based approach using SBE has not been previously reported for genotyping HHV-8. Therefore, the aim of the present study was to design and validate a SBE protocol for characterization of the HHV-8 ORFK1 subtypes.

\section{Results and discussion}

Defining the genotype of an infecting virus is important not only in epidemiology studies but also for efficient vaccine development and management of some viral infections (Venner et al. 2016; Lin and Kao 2017). However, limitations inherent to the 'gold standard' viral genotyping method, that is, phylogenetic analysis, determine that genotype assignment may not be possible in all clinical samples. Therefore, novel, faster and easier techniques for viral characterization have been proposed (Rendón et al. 2015; Nyan and Swinson 2016).

In this regard, both the SBE assay and the real-time PCR-high-resolution melting (HRM) analysis offer inexpensive, sensitive and rapid genotyping screening, need little DNA input, and have superior SNP analysis compared to (nested-) PCR and sequencing (Heideman et al. 2012). However, the source of template DNA and the way it is prepared are vitally important for the success of 
any genotyping experiment (Martín-Núnez et al. 2012) and can limit the advantages of HRM. In the case of large-scale directed genotyping by HRM curve fitting and analysis obtained from poor quality DNA isolated from FFPE tissue samples, the variability of readout leads to inadmissible noise-to-signal ratios and overlapping clustering criteria, which may lead to erroneous genotype assignment (Słomka et al. 2017), and therefore, another genotyping approach such as SBE must be chosen, as successfully applied by others (Heideman et al. 2012).

Moreover, HRM analysis is based on the comparison of melting curves that have a particular shape according to the amplicon's nucleotide composition, without determining what polymorphism is present at a specific position. This particularity makes the HRM analysis disadvantageous since it does not identify the specific polymorphism in the analysed amplicon. On the other hand, SBE technique is a method that uses extension primers that anneal to its target DNA immediately adjacent to the SNP under analysis. The fact that SBE uses specific primers for each polymorphism makes this methodology more specific in the detection of SNPs when compared to HRM analysis (Souto et al. 2015).

Recently, high-throughput next-generation sequencing (NGS) provides a large number of reads and thus coverage, to produce a reliable consensus sequence from which viral genotyping can efficiently be performed. The sequencing depth and coverage obtained from NGS would also allow to discriminate between a mutation or PCR error within the primer region or specific SNP positions and furthermore NGS data could be used to determine if any genomic recombination has occurred (Cuypers et al. 2019). Moreover, NGS has the advantage of being able to identify a wide range of potential pathogens and is able to generate a comprehensive mutational profile. However, it requires a higher workload and seems to be less sensitive compared to the standard amplification-based assays in samples with DNA fragmentation and low viral loads (Cernomaz et al. 2016; Perlejewski et al. 2020).

In addition, genome sequencing technologies are costly and not widely available in developing countries (Helmy et al. 2016) and, as a consequence, alternative genotyping methods to these genomic tools are and will still be useful in underprivileged countries.

In this study, after analysing an alignment of 76 GenBank sequences ascribed to all known HHV-8 subtypes, four SNPs were identified at positions 360, 406, 465 and 527 of the HHV-8 genome, according to GenBank accession number U75698 (Fig. 1). Thus, four primers with their $3^{\prime}$ end missing one base at these nucleotide positions were specifically designed for the multiplex SBE technique developed in this study (Table 1). Compared to ORFK1 phylogenetic analysis that requires an amplified fragment of $400-1000 \mathrm{pb}$ for reliable genotype assignment (Zong et al. 1999; Cordiali-Fei et al. 2015; Pérez and Tous 2017; Hulaniuk et al. 2020), the SBE technique described herein only needs $294 \mathrm{pb}$ for genotype characterization. This difference is particularly important when genotyping must be carried out in low-quality DNA samples, which are mostly derived from FFPE tissues (Pikor et al. 2011; Guyard et al. 2017) and commonly used in HHV-8 molecular epidemiology studies due to the particular clinical presentation of this virus. In this study, $28 \mathrm{HHV}-8$ positive FFPE samples, in which a large ORFK1 PCR product could not be amplified and thus genotyped by phylogenetic analysis, were subjected to the short-ampliconlength (294 bp) nested PCR assay described herein, and five of them $(17 \cdot 8 \%)$ could be favourably amplified and genotyped with the SBE technique (data not shown). Therefore, this method could increase the success of HHV-8 genotyping in FFPE derived samples.

In this reaction, as shown in Fig. 2, HHV-8 subtypes A-C, which are the most predominant subtypes in America, Europe, Africa and Asia (Hayward and Zong 2007), and the remaining ones (D, E and F) could be identified accurately on the basis of the four SNPs considered to be 'diagnostic' of each subtype. For example, the presence of a $\mathrm{T}$ at position 465 was characteristic of subtype $\mathrm{B}$, the detection of a $\mathrm{C}$ or a $\mathrm{T}$ at position 360 and a $\mathrm{G}$ at position 406 was distinctive of subtype $C$ and the presence of a $\mathrm{C}$ at position 527 was diagnostic of subtypes $\mathrm{D}, \mathrm{E}$ or $\mathrm{F}$ (Table 1; Fig. 2). Thus, the four patterns obtained in this reaction can be easily used to discriminate between ORFK1 subtypes (Fig. 2). However, in the case of samples belonging to subtypes $\mathrm{D}, \mathrm{E}$ and $\mathrm{F}$, the four identified SNPs can differentiate them from subtypes A-C but could not distinguish among them and, therefore, a second genotyping method or eventually a different technique is needed.

It must be highlighted that this method was successfully validated in $20 \mathrm{HHV}-8$ samples: eight belonged to subtype A, two were ascribed to subtype $B$, nine to subtype $\mathrm{C}$ and 1 belonged to subtype $\mathrm{F}$, and that the typing results obtained from SBE completely matched with those of the phylogenetic analysis in all samples (Fig. 3). Although only two samples ascribed to the African HHV-8 subtype B were tested in the validation step due to its scarce prevalence in the geographical region where this study was conducted (South America), 17 subtype $B$ sequences belonging to all known subclades (B1, B2 and B3) were randomly selected from GenBank database and their nucleotide sequence analysis confirmed that they could be identified by the four SNPs at positions 360, 406, 465 and 527 of the HHV-8 genome (Fig. 1). 


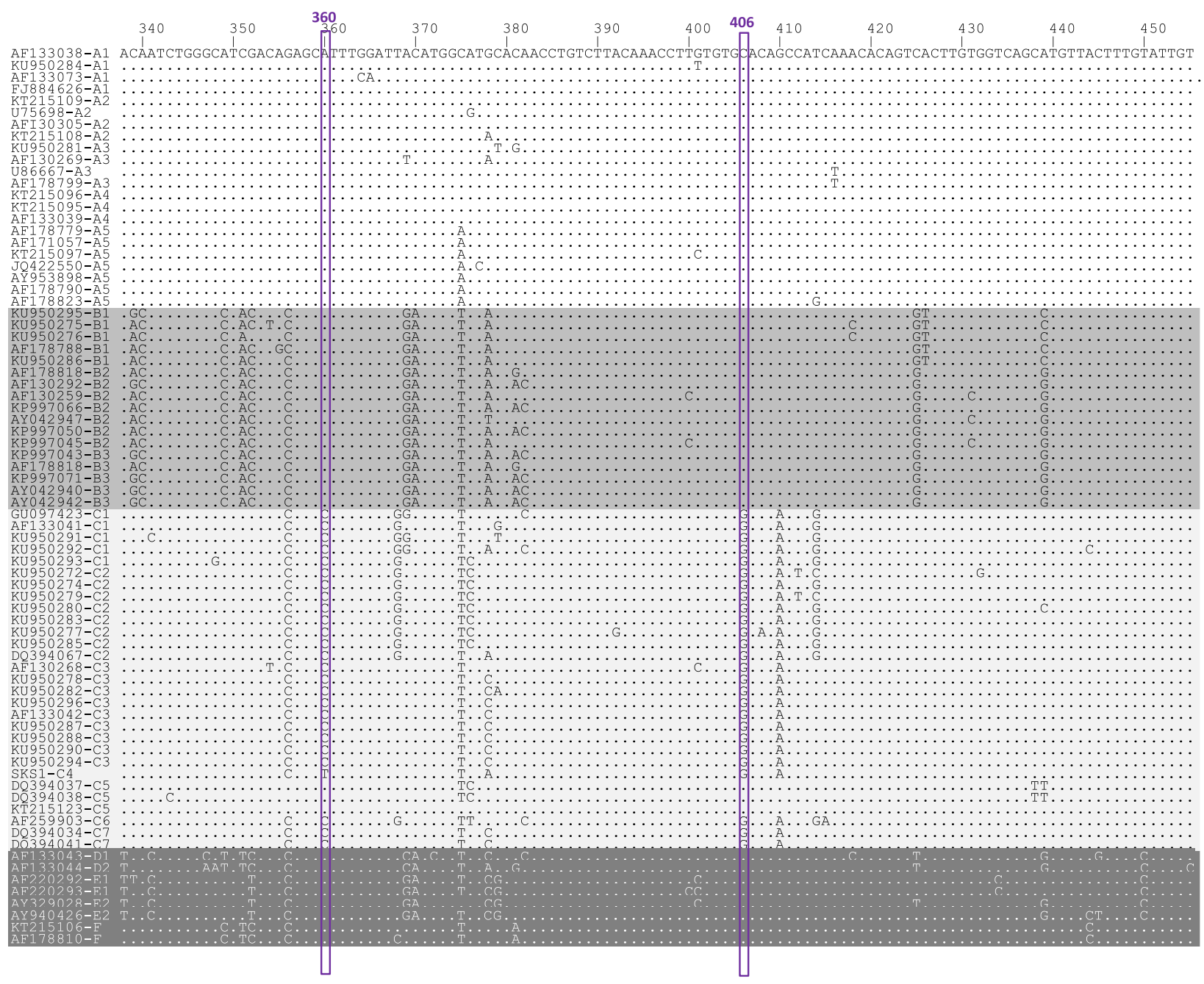

Figure 1 Alignment of partial HHV-8 ORFK1 sequences (nucleotide positions 338-532). The key nucleotides for HHV-8 genotyping are boxed. Nucleotide identity is indicated by dashes. Nucleotide positions are numbered according to GenBank accession number U75698.

Nevertheless, since genotyping is based only on four nucleotides, this technique is still prone to errors and limitations. First, PCR artefacts and errors are shared by all genotyping methods that require a PCR amplification step. In addition to the adherence to proper controls to avoid contamination, the amplification of short PCR fragments and the choice of high-fidelity DNA polymerases can reduce the probability of introducing errors in the PCR products (Zhang and Li 2003). Second, subclades in subtypes A-E could not be identified in this protocol, which could be of special interest for molecular epidemiology studies carried out in sub-Saharan Africa where subtype A5 is predominant (Mamimandjiami et al. 2021) and associated with more extensive disease in AIDS patients (Isaacs et al. 2016). Third, another limitation to this type of method is the fact that any strain harbouring mutations at these specific SNPs could alter genotype characterization (Rendón et al. 2015). Moreover, recombinants strains could not be correctly characterized. In fact, in this SBE technique, HHV-8 subtype C5 which has been identified as a $\mathrm{A} 3 / \mathrm{C} 3$ recombinant (Zong et al. 2002; Duprez et al. 2006) could be mistakenly classified as subtype A (Fig. 1). In order to achieve a higher specificity and larger geographical applicability of this SBE technique, it could be necessary to find new SNPs in another genomic region of ORFK1 that allow to discriminate subclade A5 and subtypes D-F from each other, and at the same time, sustain the results obtained from the four specific described SNPs of HHV-8.

In conclusion, since only specific SNPs useful in differentiating the HHV-8 subtypes were involved in the SBE reaction, the result analysis is easier, low-cost and rapid 


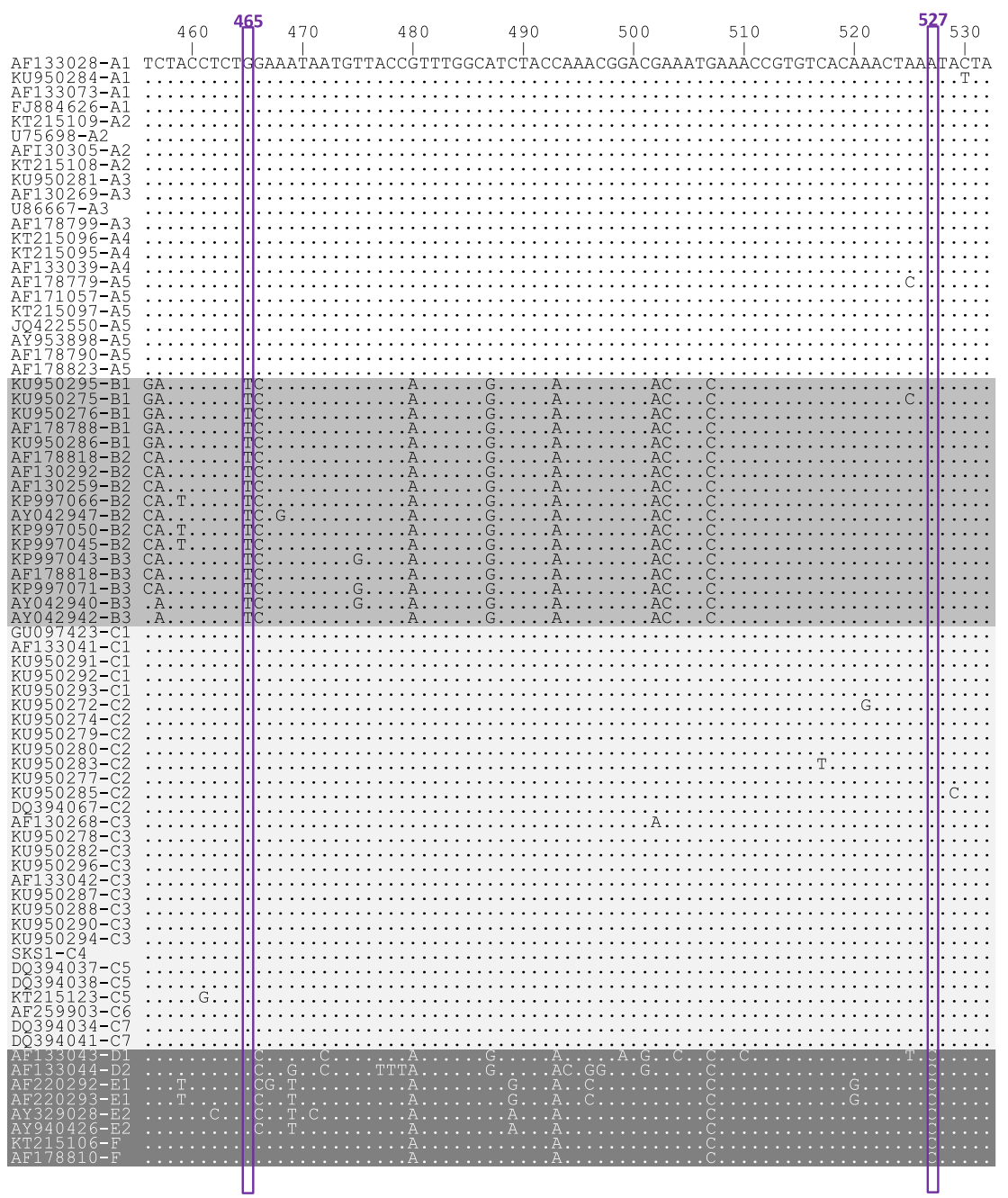

Figure 1 continued

Table 1 Primers and the nucleotides detected for HHV-8 genotyping with single base extension technique

\begin{tabular}{|c|c|c|c|c|c|c|c|}
\hline \multirow[b]{2}{*}{ Name } & \multirow[b]{2}{*}{ Nucleotide sequence $\left(5^{\prime}-3^{\prime}\right)^{*}$} & \multirow[b]{2}{*}{ Position $^{\dagger}$} & \multirow[b]{2}{*}{ SNP } & \multicolumn{4}{|c|}{ HHV-8 subtype } \\
\hline & & & & A & $\mathrm{B}$ & $\mathrm{C}$ & $D, E, F$ \\
\hline F360 & CAATCTGGGCATCGACACAGC & 339-359 & 360 & A & A & $\mathrm{C} / \pi^{+}$ & A \\
\hline F406 & ITCCTGTCTTACAAACCTTGTGTG & $384-405$ & 406 & C & $C$ & G & C \\
\hline F465 & ITTTTTTTTTTTTTTTGGTATTGTGATACCTCT & $449-464$ & 465 & G & $\mathrm{T}$ & G & G \\
\hline F527 & TTTTTTTTTTT AACCGTGTCACAAACTAA & $509-526$ & 527 & A & A & A & C \\
\hline
\end{tabular}

The nucleotides characteristics of each subtype are shown in grey.

A, adenine; C, cytosine; G, guanine; SNP, single nucleotide polymorphism; $T$, thymine.

*The $\operatorname{poly}(T)$ tails added to the $5^{\prime}$ end of each primer are underlined.

${ }^{\dagger}$ Nucleotide positions are numbered according to GenBank accession number U75698.

The nucleotide cytosine (C) is detected in all subclades of the $C$ subtype, except for subclade $C 4$.

compared to the nucleotide sequencing and phylogenetic analysis. Therefore, the SBE method described herein can be adapted to other viral genomes and be used as an efficient screening method for genotyping in a single tube, rapidly, particularly low-quality DNA samples, and with easy data interpretation. 

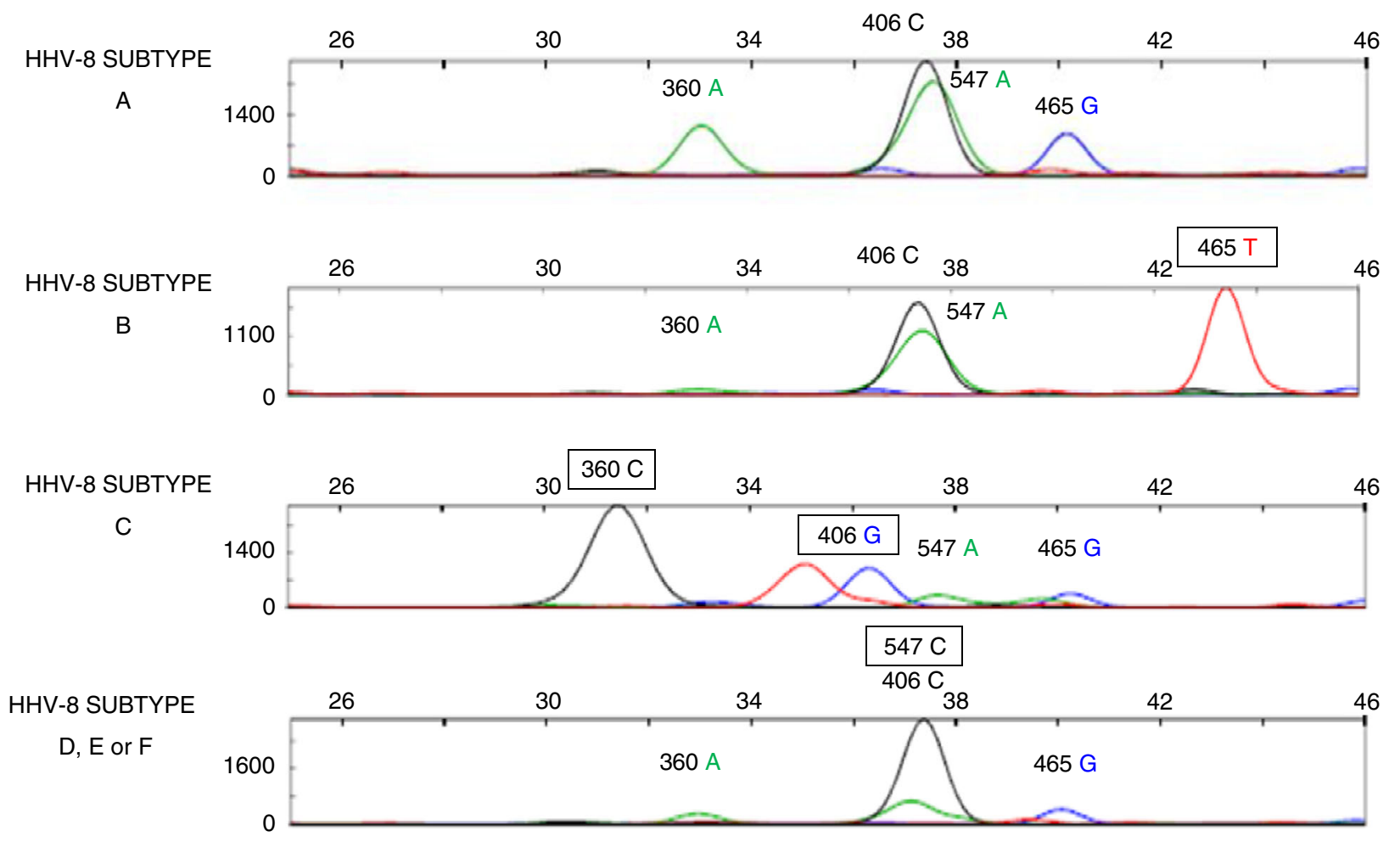

Figure 2 Multiplex single base extension electropherograms belonging to subtype A, B, C and D, E or F and analysed with GeneMapper ${ }^{\circledR}$ IDX ver. 1.4 (Thermofisher Scientific). The nucleotides detected by each one of the four single base extension primers (F360, F406, F465 and F527) are shown, and those characteristics of each subtype are boxed.

\section{Materials and methods}

DNA was extracted from 19 FFPE tissues and 1 whole blood sample from HHV-8 patients by means of FlexiGene DNA Kit or QIAamp DNA Mini Kit (Qiagen, $\mathrm{GmbH}$, Hilden, Germany) following the manufacturer's instructions. Human inosine triphosphate pyrophosphatase (ITPA) gene was amplified in all samples to test the integrity of DNA and exclude the possibility of PCR inhibitors. The presence of HHV-8 DNA was confirmed by amplification of the ORF26 as previously described (Hulaniuk et al. 2017).

Initially, nucleotide sequences retrieved from GenBank database and ascribed to HHV-8 subtypes A-F were aligned to determine SNPs that could discriminate between ORFK1 subtypes. Then, a nested PCR protocol was performed in all samples to partially amplify the ORFK1 genomic region encompassing the identified SNPs. The first amplification round amplified a 400-bp fragment with previously described primers (Ötvös et al. 2014). The reaction volume was set at $25 \mu \mathrm{l}$, and the PCR mixture consisted of $100 \mathrm{ng}$ of total DNA, $0.4 \mu \mathrm{mol}^{-1}$ of each of the $10 \mu \mathrm{mol} \mathrm{l}^{-1}$ primers, $0.2 \mathrm{mmol} \mathrm{l}^{-1}$ of
$10 \mathrm{mmol} \mathrm{l}^{-1} \mathrm{dNTPs}, 1.25 \mathrm{U}$ of GoTaq ${ }^{\circledR}$ polymerase (Promega, Madison, WI) and $1 \mathrm{X}$ of $5 \mathrm{X} \mathrm{GoTaq}{ }^{\circledR}$ reaction buffer. The PCR cycle conditions included an initial denaturation step at $95^{\circ} \mathrm{C}$ for $3 \mathrm{~min}$ and 30 amplification cycles consisting of denaturation at $95^{\circ} \mathrm{C}$ for $30 \mathrm{~s}$, annealing at $58^{\circ} \mathrm{C}$ for $60 \mathrm{~s}$ and elongation at $72^{\circ} \mathrm{C}$ for $60 \mathrm{~s}$. The second amplification round was performed with primers FW-K1 (5'-TCCTGGTATTGCAAC-3', nt 249-263) and RW-K1 (5'-CGTAAAATTATAGTA-3', nt 528-542). The PCR reaction mixture was the same as the one used for the first round, but $1 \mu \mathrm{l}$ of the product of the first round was included in the mixture. The cycling conditions of the second round included an initial denaturation step at $95^{\circ} \mathrm{C}$ for $6 \mathrm{~min}$ and 39 amplification cycles consisting of denaturation at $95^{\circ} \mathrm{C}$ for $30 \mathrm{~s}$, annealing at $60^{\circ} \mathrm{C}$ for $30 \mathrm{~s}$ and elongation at $72^{\circ} \mathrm{C}$ for $30 \mathrm{~s}$. Reactions also included a final elongation step of $5 \mathrm{~min}$ at $72^{\circ} \mathrm{C}$. PCR products were visualized after electrophoresis in $2 \%$ ethidium bromide-stained agarose gels. To test the efficacy of the short-amplicon-length (294 bp) nested PCR assay described herein, $28 \mathrm{HHV}-8$ positive FFPE samples with fragmented low-quality DNA, in which a large ORFK1 PCR product could not be amplified and thus genotyped 


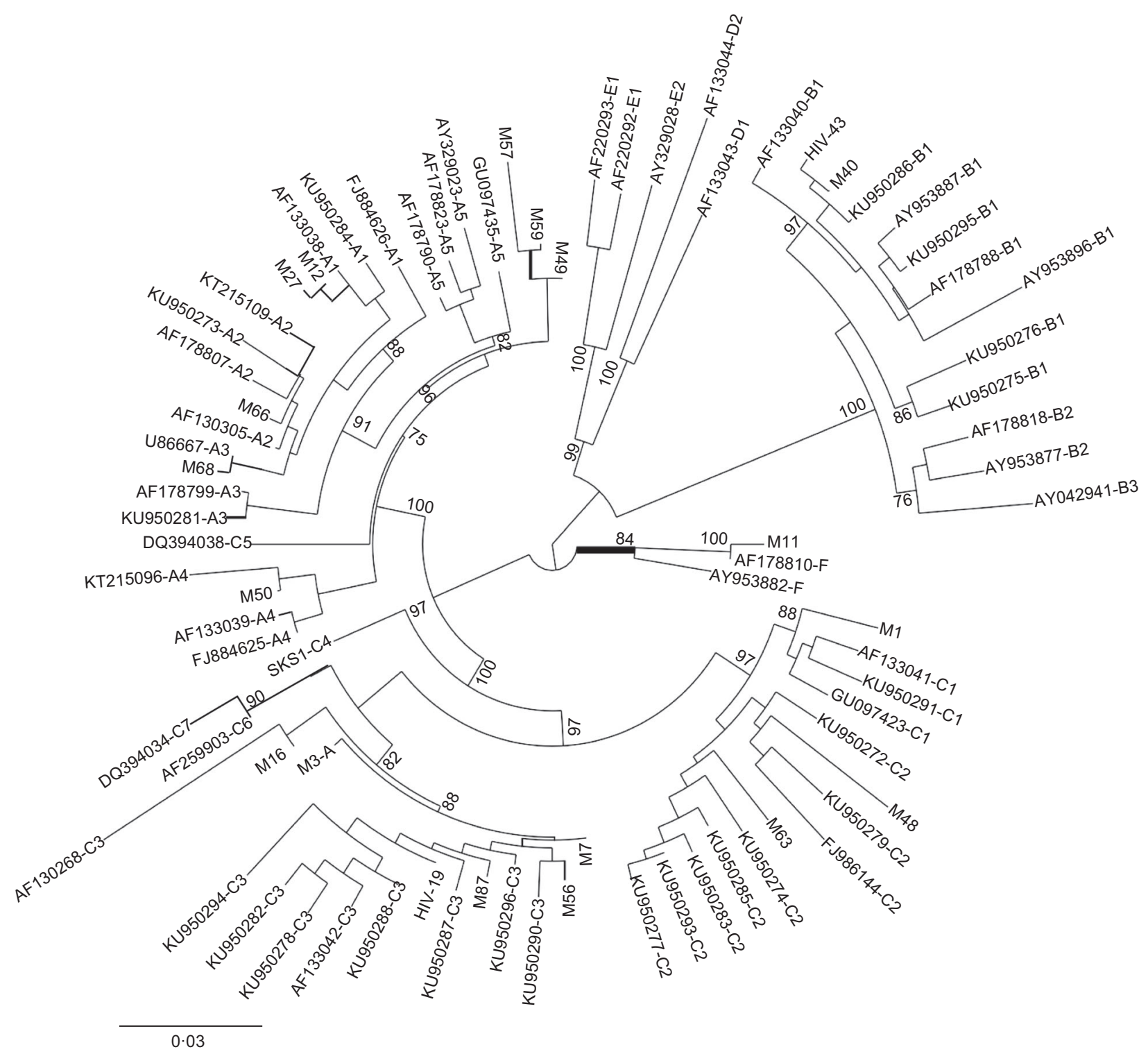

Figure 3 A phylogenetic neighbour-joining tree with Kimura's 2-parameter distance model constructed by using partial HHV-8 ORFK1 sequences (encompassing nucleotide positions 200-818) including the 20 samples in this study and 59 reference sequences from HHV-8 subtypes A-F retrieved from GenBank. The numbers at each node correspond to bootstrap values obtained with 1000 replicates (values lower than 70 are not shown). The scale bar represents the number of nucleotide substitutions per site.

by phylogenetic analysis, were subjected to this PCR protocol.

An aliquot of $4 \mu \mathrm{l}$ of the 294-bp PCR products were incubated with shrimp alkaline phosphatase and exonuclease I (Illustra ExoProStar ${ }^{\circledR}$, GE Healthcare Life Sciences, Issaquah, WA, USA) for $40 \mathrm{~min}$ at $37^{\circ} \mathrm{C}$ and then for $15 \mathrm{~min}$ at $80^{\circ} \mathrm{C}$ to remove unincorporated dNTPs and primers.

In order to detect the SNPs present in the samples during the multiplex SBE reaction, primers with their $3^{\prime}$ end missing one base at the exact nucleotide position of the discriminatory SNPs were designed using Primer3 software (http://frodo.-wi.mit.edu/prime r3). Self and heterodimers, potential hairpins and secondary structures were checked using OligoAnalyzer software (http://www.idtdna.com/annalyzer/applications/ oligoanalyzer). The primers specificity was checked using Genome Browser online software (http://genome. ucsc.edu/cgi-bin/hg). The SBE primers were $5^{\prime}$ tailed with a $\operatorname{poly}(T)$ tail of different lengths to allow a proper discrimination of the PCR fragments in the capillary electrophoresis. 
Single base extension analysis was carried out using the SNaPshot ${ }^{\circledR}$ Multiplex Ready Reaction Mix (Thermo Fisher Scientific, Waltham, MA). To tune up the reaction, first the reaction was carried out with each one of the four primers separately to check the 'diagnostic' nucleotide and then in a multiple form. The reaction was performed in a final volume of $5 \mu \mathrm{l}$ containing $0.75 \mu \mathrm{l}$ of SNaPshot ${ }^{\circledR}$ mix, $0.25 \mu \mathrm{l}$ of PCR buffer $(10 \times)$ (Thermo Fisher Scientific), $2 \cdot 5 \mu \mathrm{mol} 1^{-1}$ of F360 primer, $2 \mu \mathrm{mol} \mathrm{l}^{-1}$ of F406 primer, $2 \mu \mathrm{mol} \mathrm{l^{-1 }}$ of F465 primer, $2 \mu \mathrm{mol} \mathrm{l}^{-1}$ of $\mathrm{F} 527$ primer and $1.5 \mu \mathrm{l}$ of purified PCR products. Cycling conditions were 25 cycles of denaturation at $95^{\circ} \mathrm{C}$ for $10 \mathrm{~s}$, annealing at $50^{\circ} \mathrm{C}$ for $5 \mathrm{~s}$, and extension at $72^{\circ} \mathrm{C}$ for $4 \mathrm{~min}$. Labelled extension products were then treated with $1 \mu \mathrm{l}$ of $\mathrm{T}-\mathrm{SAP}^{\circledR}$ (Promega) for $40 \mathrm{~min}$ at $37^{\circ} \mathrm{C}$ followed by a 5 -min heat inactivation at $90^{\circ} \mathrm{C} ; 1 \mu \mathrm{l}$ of this reaction was mixed with $9.5 \mu \mathrm{l}$ of HiDi Formamide containing $0 \cdot 5 \mu \mathrm{l}$ of LIZ-120 size standard (Thermo Fisher Scientific). After denaturation at $95^{\circ} \mathrm{C}$ for $2 \mathrm{~min}$, the reaction was analysed on an ABI3100-Avant sequencer (Thermo Fisher Scientific) using GeneMapper ${ }^{\circledR}$ IDX ver. 1.4 (Thermofisher Scientific).

The HHV-8 subtype was confirmed in all samples by ORFK1 amplification and phylogenetic analysis. Briefly, ORFK1 was amplified by a previously described nested PCR protocol (Hulaniuk et al. 2020) and bidirectionally sequenced by Big-Dye Termination chemistry system (Applied Biosystem, Thermo Fisher Scientific). Multiple sequence alignments of ORFK1 sequences from the present study and reference strains reported in the GenBank were performed using BioEdit ver. 7.2.5 (Hall 1999). Phylogenetic trees were constructed using the neighbourjoining method in the MEGA 7 software (Kumar et al. 2016). Group support was evaluated by a standard bootstrap procedure (1000 pseudo-replicates). The GenBank/ EMBL/DDBJ accession numbers of the ORFK1 sequences reported in this study are: MN556696-MN556715.

This study was approved by the Ethics Committee on Research Protocols of the Italian Hospital of Buenos Aires and Muñiz Hospital in Buenos Aires, Argentina. Informed consent was obtained from the participants involved.

\section{Acknowledgements}

The authors would like to thank the patients for providing the samples used in this study. This study was supported in part by Dr. Rubén Gutman grant from the University Institute of the Italian Hospital of Buenos Aires to María Laura Hulaniuk, Bsc., and grants from the Italian Hospital of Buenos Aires to Dr. Julieta Trinks, and PIP 0914 (CONICET) and UBACyT 20020130100783BA to Dr. Daniel Corach.

\section{Author contribution}

All authors listed contributed to this work and are entitled to authorship. Dr. Caputo directed and coordinated this study. Dr. Corach, Dr. Trinks and Dr. Caputo provided conceptual and technical guidance for all the aspects of the project. Dr. Hulaniuk and Dr. Trinks recruited HHV-8 patients and collected the biological samples. Dr. Hulaniuk designed the SBE primers and conducted the performance testing of the technique. Dr. Hulaniuk and Dr. Caputo analysed the data. Dr. Trinks drafted the manuscript. All authors were involved in manuscript editing and approved the version submitted for publication.

\section{Conflict of Interest}

No conflict of interest declared.

\section{REFERENCES}

Abecasis, A.B., Pingarilho, M. and Vandamme, A.M. (2018) Phylogenetic analysis as a forensic tool in HIV transmission investigations. AIDS 32, 543-554.

Araujo, R., Eusebio, N. and Caramalho, R. (2015) Feasibility of mini-sequencing schemes based on nucleotide polymorphisms for microbial identification and population analyses. Appl Microbiol Biotechnol 99, 2513-2521.

Arpan, A., Salil, V., Harsh, P. and Pratap, N.M. (2016) Single base primer extension assay (SNaPshot) for rapid detection of human immunodeficiency virus -1 Drug resistance mutations. J Mol Biomark Diagn 7, 2.

Bons, E. and Regoes, R.R. (2018) Virus dynamics and phyloanatomy: merging population dynamic and phylogenetic approaches. Immunol Rev 285, 134-146.

Cassar, O., Charavay, F., Bassot, S., Plancoulaine, S., Grangeon, J.P., Laumond-Barny, S., Martin, P.M.V., Chanteau, S. et al. (2012) Divergent KSHV/HHV-8 subtype D strains in New Caledonia and Solomon Islands, Melanesia. J Clin Virol 53, 214-218.

Cella, E., Riva, E., Angeletti, S., Fogolari, M., Blasi, A., Scolamacchia, V., Spoto, S., Bazzardi, R. et al. (2018) Genotype I hepatitis A virus introduction in Italy: Bayesian phylogenetic analysis to date different epidemics. J Med Virol 90, 1493-1502.

Cernomaz, A.-T., Macovei, I.I., Pavel, I., Grigoriu, C., Marinca, M., Baty, F., Peter, S., Zonda, R. et al. (2016) Comparison of next generation sequencing, SNaPshot assay and real-time polymerase chain reaction for lung adenocarcinoma EGFR mutation assessment. BMC Pulm Med 16, 88.

Chen, D.P., Wen, Y.H., Lu, J.J., Tseng, C.P. and Wang, W.T. (2019) Rapid rare ABO blood typing using a single PCR based on a multiplex SNaPshot reaction. J Formos Med Assoc 118, 395-400. 
Ciotti, M., Angeletti, S., Minieri, M., Giovannetti, M., Benvenuto, D., Pascarella, S., Sagnelli, C., Bianchi, M. et al. (2019) COVID-19 outbreak: an overview. Chemotherapy 64, 215-223.

Cordiali-Fei, P., Trento, E., Giovanetti, M., Lo Presti, A., Latini, A., Giuliani, M., D’Agosto, G., Bordignon, V. et al. (2015) Analysis of the ORFK1 hypervariable regions reveal distinct HHV-8 clustering in Kaposi's sarcoma and nonKaposi's cases. J Exp Clin Cancer Res 34, 1.

Cuypers, L., Thijssen, M., Shakibzadeh, A., Sabahi, F., Ravanshad, M. and Pourkarim, M.R. (2019) Nextgeneration sequencing for the clinical management of hepatitis $\mathrm{C}$ virus infections: does one test fits all purposes? Crit Rev Clin Lab Sci 56, 420-434.

Do, H. and Dobrovic, A. (2015) Sequence artifacts in DNA from formalin-fixed tissues: causes and strategies for minimization. Clin Chem 61, 64-71.

Duprez, R., Hbid, O., Afonso, P., Quach, H., Belloul, L., Fajali, N., Ismaili, N., Benomar, H. et al. (2006) Molecular epidemiology of the HHV-8 K1 gene from Moroccan patients with Kaposi's sarcoma. Virology 353, 121-132.

Ganesh, A. and Zhang, Q. (2019) Optimal sequence length requirements for phylogenetic tree reconstruction with indels. In STOC 2019: Proceedings of the 51st Annual ACM SIGACT Symposium on Theory of Computing ed. Charikar, M. and Cohen, E.. pp. 721-732. New York, NY: Association for Computing Machinery.

Guyard, A., Boyez, A., Pujals, A., Robe, C., Tran Van Nhieu, J., Allory, Y., Moroch, J., Georges, O. et al. (2017) DNA degrades during storage in formalin-fixed and paraffinembedded tissue blocks. Virchows Arch 471, 491-500.

Hall, T.A. (1999) BioEdit: a user-friendly biological sequence alignment editor and analysis program for windows 95/98/ NT. Nucleic Acids Symp Ser 41, 95-98.

Hayward, G.S. and Zong, J.C. (2007) Modern evolutionary history of the human KSHV genome. Curr Top Microbiol Immunol 312, 1-42.

Heideman, D.A.M., Lurkin, I., Doeleman, M., Smit, E.F., Verheul, H.M., Meijer, G.A., Snijders, P.J.F., Thunnissen, E. et al. (2012) KRAS and BRAF mutation analysis in routine molecular diagnostics: comparison of three testing methods on formalin-fixed, paraffin-embedded tumorderived DNA. J Mol Diagn 14, 247-255.

Helmy, M., Awad, M. and Mosa, K.A. (2016) Limited resources of genome sequencing in developing countries: challenges and solutions. Appl Transl Genom 9, 15-19.

Hulaniuk, M.L., Mojsiejczuk, L., Jauk, F., Remondegui, C., Mammana, L., Bouzas, M.B., Zapiola, I., Ferro, M.V. et al. (2020) Genetic diversity and phylogeographic analysis of human herpesvirus type 8 (HHV-8) in two distant regions of Argentina: association with the genetic ancestry of the population. Infect Genet Evol 85, 104523.

Hulaniuk, M.L., Torres, O., Bartoli, S., Fortuny, L., Burgos Pratx, L., Nuñez, F., Salamone, H., Corach, D. et al.
(2017) Increased prevalence of human herpesvirus type 8 (HHV-8) genome among blood donors from NorthWestern Argentina. J Med Virol 89, 518-527.

Irshad, M., Gupta, P., Mankotia, D.S. and Ansari, M.A. (2016) Multiplex qPCR for serodetection and serotyping of hepatitis viruses: a brief review. World J Gastroenterol 22, 4824-4834.

Isaacs, T., Abera, A.B., Muloiwa, R., Katz, A.A. and Todd, G. (2016) Genetic diversity of HHV8 subtypes in South Africa: A5 subtype is associated with extensive disease in AIDS-KS. J Med Virol 88, 292-303.

Jary, A., Leducq, V., Desire, N., Petit, H., Palich, R., Joly, V., Canestri, A., Gothland, A. et al. (2020) New Kaposi's sarcoma-associated herpesvirus variant in men who have sex with men associated with severe pathologies. J Infect Dis 222, 1320-1328.

Kajumbula, H., Wallace, R.G., Zong, J.-C., Hokello, J., Sussman, N., Simms, S., Rockwell, R.F., Pozos, R. et al. (2006) Ugandan Kaposi's sarcoma-associated herpesvirus phylogeny: evidence for cross-ethnic transmission of viral subtypes. Intervirology 49, 133-143.

Kenmoe, S., Vernet, M.A., Penlap Beng, V., Vabret, A. and Njouom, R. (2020) Phylogenetic variability of Human Metapneumovirus in patients with acute respiratory infections in Cameroon, 2011-2014. J Infect Public Health 13, 606-612.

Kocjan, B.J., Hošnjak, L. and Poljak, M. (2016) Detection of alpha human papillomaviruses in archival formalin-fixed, paraffin-embedded (FFPE) tissue specimens. J Clin Virol 76, S88-S97.

Kumar, S., Stecher, G. and Tamura, K. (2016) MEGA7: molecular evolutionary genetics analysis version 7.0 for bigger datasets. Mol Biol Evol 33, 1870-1874.

Li, S., Bai, L., Dong, J., Sun, R. and Lan, K. (2017) Kaposi's sarcoma-associated herpesvirus: epidemiology and molecular biology. Adv Exp Med Biol 1018, 91-127.

Lin, C.L. and Kao, J.H. (2017) Natural history of acute and chronic hepatitis B: the role of HBV genotypes and mutants. Best Pract Res Clin Gastroenterol 31, 249-255.

Mamimandjiami, A.I., Mouinga-Ondémé, A., Ramassamy, J.L., Djuicy, D.D., Afonso, P.V., Mahé, A., Lekana-Douki, J.B., Cassar, O. et al. (2021) Epidemiology and genetic variability of $\mathrm{HHV}-8 / \mathrm{KSHV}$ among rural populations and Kaposi's sarcoma patients in Gabon, Central Africa. Review of the geographical distribution of HHV-8 K1 genotypes in Africa. Viruses 13, 175.

Mancuso, R., Biffi, R., Valli, M., Bellinvia, M., Athanasia, T., Ferrucci, S., Brambilla, L., Delbue, S. et al. (2008) HHV8 a subtype is associated with rapidly evolving classic Kaposi's sarcoma. J Med Virol 80, 2153-2160.

Marashi, S.M., Mostafa, A., Shoja, Z., Nejati, A., Shahmahmoodi, S., Mollaei-Kandelous, Y., Sahraian, M.A. and Jalilvand, S. (2018) Human herpesvirus 8 DNA detection and variant analysis in patients with multiple sclerosis. Virusdisease 29, 540-543. 
Martín-Núñez, G.M., Gómez-Zumaquero, J.M., Soriguer, F. and Morcillo, S. (2012) High resolution melting curve analysis of DNA samples isolated by different DNA extraction methods. Clin Chim Acta 413, 331-333.

Mou, X., Sheng, D., Chen, Z., Liu, M., Liu, Y., Deng, Y., Xu, K., Hou, R. et al. (2019) In-situ mutation detection by magnetic beads-probe based on single base extension and its application in genotyping of hepatitis B virus pre-C region 1896 nt locus single nucleotide polymorphisms. $J$ Biomed Nanotechnol 15, 2393-2400.

Mubemba, B., Thompson, P.N., Odendaal, L., Coetzee, P. and Venter, E.H. (2017) Evaluation of positive Rift Valley fever virus formalin-fixed paraffin embedded samples as a source of sequence data for retrospective phylogenetic analysis. J Virol Methods 243, 10-14.

Naidu, H., Subramanian, B.M., Chinchkar, S.R., Sriraman, R., Rana, S.K. and Srinivasan, V.A. (2012) Typing of canine parvovirus isolates using mini-sequencing based single nucleotide polymorphism analysis. J Virol Methods 181, 197-201.

Nyan, D.C. and Swinson, K.L. (2016) A method for rapid detection and genotype identification of hepatitis $\mathrm{C}$ virus $1-6$ by one-step reverse transcription loopmediated isothermal amplification. Int J Infect Dis 43, 30-36.

Ötvös, R., Juhasz, A., Szalai, E., Ujvari, D., Ötvös, K., Szabo, K., Remenyik, E., Szekely, L. et al. (2014) Molecular typing of human herpesvirus 8 isolates from patients with Kaposi's sarcoma in Hungary. Anticancer Res 34, 893-898.

Peng, D., Zhang, Y., Ren, H., Li, H., Li, R., Shen, X., Wang, N., Huang, E. et al. (2020) Identification of sequence polymorphisms at 58 STRs and 94 iiSNPs in a Tibetan population using massively parallel sequencing. Sci Rep 10, 12225.

Pérez, C.L. and Tous, M.I. (2017) Diversity of human herpesvirus 8 genotypes in patients with AIDS and nonAIDS associated Kaposi's sarcoma, Castleman's disease and primary effusion lymphoma in Argentina. J Med Virol 89, 2020-2028.

Perlejewski, K., Bukowska-Ośko, I., Rydzanicz, M., Pawełczyk, A., Caraballo Cortès, K., Osuch, S., Paciorek, M., Dzieciątkowski, T. et al. (2020) Next-generation sequencing in the diagnosis of viral encephalitis: sensitivity and clinical limitations. Sci Rep 10, 16173.

Pikor, L.A., Enfield, K.S., Cameron, H. and Lam, W.L. (2011) DNA extraction from paraffin embedded material for genetic and epigenetic analyses. J Vis Exp 49, 2763.

Ranjbar, R., Karami, A., Farshad, S., Giammanco, G.M. and Mammina, C. (2014) Typing methods used in the molecular epidemiology of microbial pathogens: a how-to guide. New Microbiol 37, 1-15.

Rendón, J.C., Cortés-Mancera, F., Duque-Jaramillo, A., Ospina, M.C. and Navas, M.C. (2015) Analysis of hepatitis B virus genotypes by restriction fragment length polymorphism. Biomedica 36, 79-88.
Słomka, M., Sobalska-Kwapis, M., Wachulec, M., Bartosz, G. and Strapagiel, D. (2017) High resolution melting (HRM) for high-throughput genotyping-limitations and caveats in practical case studies. Int J Mol Sci 18, 2316.

Sobrino, B., Brión, M. and Carracedo, A. (2005) SNPs in forensic genetics: a review on SNP typing methodologies. Forensic Sci Int 154, 181-194.

Souto, L., Tavares, F., Moreira, H. and Pinheiro, F. (2015) A comparison of SNaPshot minisequencing and HRM analysis in mtSNP genotyping with reference samples from east Timor. J Genet Genome Res 2, 021.

Tozetto-Mendoza, T.R., Ibrahim, K.Y., Tateno, A.F., de Oliveira, C.M., Sumita, L.M., Sanchez, M.C.A., Luna, E.J., Pierrotti, L.C. et al. (2016) Genotypic distribution of HHV-8 in AIDS individuals without and with Kaposi sarcoma: Is genotype B associated with better prognosis of AIDS-KS? Medicine (Baltimore) 95, e5291.

Venner, C.M., Nankya, I., Kyeyune, F., Demers, K., Kwok, C., Chen, P.-L., Rwambuya, S., Munjoma, M. et al. (2016) Infecting HIV-1 subtype predicts disease progression in women of Sub-Saharan Africa. EBioMedicine 13, 305-314.

Webster, D.R., Hekele, A.G., Lauring, A.S., Fischer, K.F., Li, H., Andino, R. and DeRisi, J.L. (2009) An enhanced single base extension technique for the analysis of complex viral populations. PLoS ONE 4, e7453.

Wohl, S., Schaffner, S.F. and Sabeti, P.C. (2016) Genomic analysis of viral outbreaks. Annu Rev Virol 3, 173-195.

Young, A.D. and Gillung, J.P. (2019) Phylogenomics principles, opportunities and pitfalls of big-data phylogenetics. Syst Entomol 45, 225-247.

Zeddeman, A., Witteveen, S., Bart, M.J., van Gent, M., van der Heide, H.G., Heuvelman, K.J., Schouls, L.M. and Mooi, F.R. (2015) Studying Bordetella pertussis populations by use of SNPeX, a simple high-throughput single nucleotide polymorphism typing method. J Clin Microbiol 53, 838846.

Zhang, D., Pu, X., Wu, W., Jin, Y., Juhear, M. and Wu, X. (2008) Genotypic analysis on the ORF-K1 gene of human herpesvirus 8 from patients with Kaposi's sarcoma in Xinjiang, China. J Genet Genomics 35, 657-663.

Zhang, J. and Li, K. (2003) Single-base discrimination mediated by proofreading $3^{\prime}$ phosphorothioate-modified primers. Mol Biotechnol 25, 223-227.

Zong, J.-C., Ciufo, D.M., Alcendor, D.J., Wan, X., Nicholas, J., Browning, P.J., Rady, P.L., Tyring, S.K. et al. (1999) Highlevel variability in the ORF-K1 membrane protein gene at the left end of the Kaposi's sarcoma-associated herpesvirus genome defines four major virus subtypes and multiple variants or clades in different human populations. J Virol 73, 4156-4170.

Zong, J., Ciufo, D.M., Viscidi, R., Alagiozoglou, L., Tyring, S., Rady, P., Orenstein, J., Boto, W. et al. (2002) Genotypic analysis at multiple loci across Kaposi's sarcoma herpesvirus (KSHV) DNA molecules: clustering patterns, novel variants and chimerism. J Clin Virol 23, 119-148. 